PROCEEDINGS OF THE

AMERICAN MATHEMATICAL SOCIETY

Volume 128, Number 4, Pages 1109-1116

S 0002-9939(99)05103-5

Article electronically published on August 17, 1999

\title{
BOUNDED COMPOSITION OPERATORS WITH CLOSED RANGE ON THE DIRICHLET SPACE
}

\author{
DANIEL H. LUECKING
}

(Communicated by Albert Baernstein II)

\begin{abstract}
For composition operators on spaces of analytic functions it is well known that norm estimates can be converted to Carleson measure estimates. The boundedness of the composition operator becomes equivalent to a Carleson measure inequality. The measure corresponding to a composition operator $C_{\varphi}$ on the Dirichet space $\mathcal{D}$ is $d \nu_{\varphi}=n_{\varphi} d A$, where $n_{\varphi}(z)$ is the cardinality of the preimage $\varphi^{-1}(z)$. The composition operator will have closed range if and only if the corresponding measure satisfies a "reverse Carleson measure" theorem: $\|f\|_{\mathcal{D}}^{2} \leq \int\left|f^{\prime}\right|^{2} d \nu_{\varphi}$ for all $f \in \mathcal{D}$. Assuming $C_{\varphi}$ is bounded, a necessary condition for this inequality is a reverse of the Carleson condition: (C) $\nu_{\varphi}(S) \geq c|S|$ for all Carleson squares $S$. It has long been known that this is not sufficient for a completely general measure. Here we show that it is also not sufficient for the special measures $\nu_{\varphi}$. That is, we construct a function $\varphi$ such that $C_{\varphi}$ is bounded and $\nu_{\varphi}$ satisfies (C) but the composition operator $C_{\varphi}$ does not have closed range.
\end{abstract}

\section{INTRODUCTION}

Let $\varphi$ be an analytic map from the unit disk $\mathbb{D}$ into itself. For any analytic function $f$ on $\mathbb{D}$ define $C_{\varphi}(f)=f \circ \varphi$. This defines a linear transformation known as a composition operator. Let $d A$ be area measure on the unit disk and let $\mathcal{D}$ denote the Dirichlet space of analytic functions on $\mathbb{D}$ satisfying $\int_{\mathbb{D}}\left|f^{\prime}\right|^{2} d A<\infty$. We give $\mathcal{D}$ the norm $\|f\|_{\mathcal{D}}^{2}=|f(0)|^{2}+\int\left|f^{\prime}\right|^{2} d A$. There is a well know necessary and sufficient condition for $C_{\varphi}$ to be bounded on $\mathcal{D}$. It arises from the following observation:

$$
\int_{\mathbb{D}}\left|(f \circ \varphi)^{\prime}\right|^{2} d A=\int_{\mathbb{D}}\left|f^{\prime}(\varphi)\right|^{2}\left|\varphi^{\prime}\right|^{2} d A=\int\left|f^{\prime}\right|^{2} n_{\varphi} d A
$$

where $n_{\varphi}(w)$ is the cardinality of the set $\varphi^{-1}(w)$. The boundedness of $C_{\varphi}$ is equivalent to $n_{\varphi} d A$ being a Carleson measure for the Bergman space. Let $\psi(z, w)=$ $|z-w| /|1-\bar{z} w|$ denote the pseudohyperbolic metric, and for $0<\eta<1$, let $D_{\eta}(w)=\{z \in \mathbb{D}: \psi(z, w)<\eta\}$. The condition for $n_{\varphi} d A$ to be a Carleson measure for the Bergman space is that there exist a constant $C$ independent of $z$

Received by the editors February 23, 1998 and, in revised form, June 1, 1998.

1991 Mathematics Subject Classification. Primary 46E20.

Key words and phrases. Composition operator, closed range. 
such that for all (some) $0<\eta<1$

$$
\int_{D_{\eta}(z)} n_{\varphi} d A \leq C\left|D_{\eta}(z)\right| \text { for all } z \in \mathbb{D} .
$$

Here $\left|D_{\eta}(z)\right|$ denotes the area of $D_{\eta}(z)$.

For any $\zeta \in \partial \mathbb{D}$ and $0<h<2$ let $S(\zeta, h)=\{z \in \mathbb{D}:|z-\zeta|<h\}$. Equivalent to (1.1) is the following condition:

$$
\int_{S(\zeta, h)} n_{\varphi} d A \leq C|S(\zeta, h)| \text { for all } \zeta \in \partial \mathbb{D} \text { and all } 0<h<2 .
$$

Of course, the constants $C$ in (1.1) and (1.2) need not be the same.

We are concerned with the condition that $C_{\varphi}: \mathcal{D} \rightarrow \mathcal{D}$ have closed range as well as being bounded. After the usual accomodation for the $|f(0)|^{2}$ term in $\|f\|_{\mathcal{D}}^{2}$ (see [2]), the necessary and sufficient condition for this is that

$$
\frac{1}{C} \int\left|f^{\prime}\right|^{2} d A \leq \int\left|f^{\prime}\right|^{2} n_{\varphi} d A \leq C \int\left|f^{\prime}\right|^{2} d A
$$

for some constant $C$ and all $f \in \mathcal{D}$. A necessary condition for this (see [3]) is condition (1.1) plus the following:

$$
\text { for some } 0<R<1 \text { and } \delta>0 \quad \int_{D_{R}(z)} n_{\varphi} d A \geq \delta\left|D_{R}(z)\right| \text { for all } z \in \mathbb{D} \text {, }
$$

or the equivalent version

$$
\text { for some } \delta>0 \quad \int_{S(\zeta, h)} n_{\varphi} d A \geq \delta|S(\zeta, h)| \quad \text { for all } S(\zeta, h) \text {. }
$$

I and others have conjectured that this necessary condition might be sufficient. The condition in [3] on general Carleson measures (on which condition (1.4) is based) is not in general a sufficient condition for that setting. However, the best counterexamples of that sufficiency were discrete measures concentrated on a zero set for the Bergman space and for a while it seemed possible that measures of the form $n_{\varphi} d A$ might behave unlike discrete measures. Then the results in 4 indicated perhaps the opposite, but I found it difficult to construct functions $\varphi$ to make $n_{\varphi} d A$ have the required properties. The examples constructed in 7$]$ and expanded on in 2] provided the tools necessary to produce a counterexample. The rest of this paper is involved in constructing an analytic map $\varphi$ from $\mathbb{D}$ to itself which satisfies conditions (1.1) and (1.4) but $C_{\varphi}$ does not have closed range. Thus, conditions (1.1) and (1.4) are not sufficient for $C_{\varphi}$ to have closed range. Section 2 presents some of the main ideas about Carleson measures that will be needed. Lemmas 2.2 and 2.4

isolate the goals of the construction. Section 3 contains the actual construction of $\varphi$.

\section{Preliminaries on Carleson measures}

The Bergman space $A^{p}$ is defined for any $p>0$ as the space of all analytic functions on $\mathbb{D}$ such that the 'norm'

$$
\|f\|_{p}=\left(\int_{\mathbb{D}}|f|^{p} d A\right)^{1 / p}
$$


is finite. A Carleson measure for $A^{p}$ is a positive measure $\mu$ on $\mathbb{D}$ satisfying

$$
\int|f|^{p} d \mu \leq C\|f\|_{p}^{p} \quad \text { for all } f \in A^{p}
$$

The general form of condition (1.1) provides a necessary and sufficient condition for a measure $\mu$ to be a Carleson measure:

$$
\|\mu\|_{*} \stackrel{\text { def }}{=} \sup _{z \in \mathbb{D}} \frac{\mu\left(D_{\eta}(z)\right)}{\left|D_{\eta}(z)\right|}<\infty .
$$

Note that we do not indicate the dependency on $\eta$ in the definition of the norm $\|\mu\|_{*}$. Different values of $\eta$ produce equivalent norms, and the value of $\eta$ will always be fixed within any discussion involving this norm.

If $\mu$ is a Carleson measure for $A^{p}$, then the identity operator is continuous from $A^{p}$ to $L^{p}(\mu)$. The condition for this operator to be an isomorphic embedding is

$$
\text { for some constant } C, \quad \int_{\mathbb{D}}|f|^{p} d A \leq C \int|f|^{p} d \mu \quad \text { for all } f \in A^{p} \text {. }
$$

In 3] it was shown that in the presence of condition (2.2), a necessary condition for (2.3) is

$$
\text { for some } 0<R<1 \text { and } \delta>0 \quad \mu\left(D_{R}(z)\right)>\delta\left|D_{R}(z)\right| \quad \text { for all } z \in \mathbb{D} .
$$

This is the source of condition (1.4): the set of derivatives of functions in $\mathcal{D}$ is $A^{2}$ and the measure in question is $d \mu=n_{\varphi} d A$.

An example showing that (2.4) is not sufficient is easily obtained by constructing an ' $(\eta, R)$-lattice' which is a zero set for $A^{p}$. An $(\eta, R)$-lattice is a sequence $\left\{z_{k}\right\}$ in $\mathbb{D}$ such that the disks $D_{\eta}\left(z_{k}\right)$ are disjoint and the disks $D_{R}\left(z_{k}\right)$ cover $\mathbb{D}$. Such a sequence is a zero sequence for a function in $A^{p}$ provided $\eta$ is sufficiently near 1 (see [1, [5] and [6]). Construct a measure $\mu$ to have mass $\left|D_{\eta}\left(z_{k}\right)\right|$ concentrated at each point $z_{k}$. It is easily seen that (2.2) is satisfied and (2.4) is satisfied with $\delta=$ $\inf \left(\left|D_{\eta}\left(z_{k}\right)\right| /\left|D_{R}(w)\right|\right)>0$, the infimum being taken over all $k$ and all $w \in D_{R}\left(z_{k}\right)$. However, (2.3) fails for the functions $f$ having zeros at all $z_{k}$. We will later show that a measure need only be concentrated "near" a zero set in an appropriate sense, for (2.3) to fail.

A measure $\nu$ is called a vanishing Carleson measure if for every $0<\eta<1$

$$
\sup _{r<|z|<1} \frac{\nu\left(D_{\eta}(z)\right)}{\left|D_{\eta}(z)\right|} \rightarrow 0 \quad \text { as } r \rightarrow 1 .
$$

These are the measures such that the identity map from $A^{p}$ to $L^{p}(\nu)$ is compact.

In 3 ] it was pointed out that the implication (2.2) $\Rightarrow(2.1)$ could be improved to the following using the same proof:

Lemma 2.1. Let $\mu$ be a positive measure on $\mathbb{D}$ that satisfies condition (2.2). Let $E$ be any Borel set in $\mathbb{D}$ and $E_{\eta}$ denote the set of points with pseudohyperbolic distance from $E$ less than $\eta$ (same $\eta$ as in (2.2)). There is a constant $C$ depending only on $\eta$ such that if $f$ is analytic in $\mathbb{D}$

$$
\int_{E}|f|^{p} d \mu \leq C\left\|\left.\mu\right|_{E}\right\|_{*} \int_{E_{\eta}}|f|^{p} d A
$$

Two things to note about (2.5): the constant $C$ is independent of $\mu$ and the integral on the right could be taken on quite a small subset of $\mathbb{D}$. Note that a vanishing Carleson measure $\nu$ is one satisfying $\left\|\left.\nu\right|_{A_{r}}\right\|_{*} \rightarrow 0$ as $r \rightarrow 1$, where $A_{r}$ 
denotes the annulus $r<|z|<1$. This observation makes the following almost obvious.

Lemma 2.2. If $\nu$ is a vanishing Carleson measure and $f \in A^{p}$, then

$$
\lim _{m \rightarrow \infty} \frac{\int\left|z^{m} f(z)\right|^{p} d \nu(z)}{\int\left|z^{m} f(z)\right|^{p} d A(z)}=0
$$

Proof. Select any $\epsilon>0$ and choose $0<r<1$ so near to 1 that

$$
\int_{A_{r}}|g(z)|^{p} d \nu(z) \leq \frac{\epsilon}{2} \int|g(z)|^{p} d A(z)
$$

for all $g \in A^{p}$. This holds, in particular, for all $g$ of the form $z^{m} f(z)$ and $r$ is independent of $m$. It remains to show that for sufficiently large $m$

$$
\int_{D_{r}(0)}\left|z^{m} f(z)\right|^{p} d \nu(z) \leq(\epsilon / 2) \int\left|z^{m} f(z)\right|^{p} d A(z) .
$$

Note that if $E=D_{r}(0)=\{z:|z|<r\}$, then $E_{\eta}=D_{s}(0)=\{|z|<s\}$ with $r<s=(r+\eta) /(1+r \eta)<1$. By Lemma 2.1

$$
\begin{aligned}
\int_{D_{r}(0)}\left|z^{m} f(z)\right|^{p} d \nu(z) & \leq C\|\nu\|_{*} \int_{D_{s}(0)}\left|z^{m} f(z)\right|^{p} d A(z) \\
& \leq C\|\nu\|_{*} s^{m p} \int_{D_{s}(0)}|f(z)|^{p} d A(z) .
\end{aligned}
$$

Now the integrals $\int_{0}^{2 \pi}\left|f\left(r e^{i \theta}\right)\right|^{p} d \theta$ are increasing functions of $r$, so their average over $[0, s]$ with respect to the measure $r d r$ is less than their average over $[t, 1]$ if $s<t<1$ :

$$
\frac{1}{s^{2}} \int_{D_{s}(0)}|f(z)|^{p} d A(z) \leq \frac{1}{1-t^{2}} \int_{A_{t}}|f(z)|^{p} d A(z) .
$$

Fix any such $t$ to obtain

$$
\int_{D_{r}(0)}\left|z^{m} f(z)\right|^{p} d \nu(z) \leq C\|\nu\|_{*} \frac{s^{m p+2}}{1-t^{2}} \int_{A_{t}}|f(z)|^{p} d A(z) .
$$

Finally, because $\int_{A_{t}}|f(z)|^{p} d A(z) \leq t^{-m p} \int\left|z^{m} f(z)\right|^{p} d A(z)$ we get

$$
\int_{D_{r}(0)}\left|z^{m} f(z)\right|^{p} d \nu(z) \leq C\|\nu\|_{*} \frac{s^{m p+2}}{t^{m p}\left(1-t^{2}\right)} \int\left|z^{m} f(z)\right|^{p} d A(z) .
$$

Now since $s^{m} / t^{m} \rightarrow 0$ as $m \rightarrow \infty$, simply choose $m$ so large that the expression preceding the integral is less than $\epsilon / 2$.

It was already clear that a vanishing Carleson measure cannot satisfy (2.3): it doesn't satisfy the necessary condition (2.4). But Lemma2.2] shows something more that we need later: that for any fixed $f \in A^{p}$ it cannot satisfy (2.3) on any sequence of functions of the form $z^{m} f$. The proof actually shows that the convergence of the limit to zero is uniform in $f$, but we will not need this fact.

Lemma 2.3. Let $\left\{z_{k}\right\}$ be a sequence of points such that the disks $D_{\eta}\left(z_{k}\right)$ are disjoint, and assume that $f \in A^{p}$ vanishes at all the points $z_{k}$. Let $\mu$ be a Carleson 
measure for $A^{p}$ which vanishes off the union $\bigcup_{k} D_{\delta}\left(z_{k}\right)$ for some $\delta<\eta / 2$. Then there is a constant $C$ depending only on $\eta$ and $p$ such that

$$
\int|f|^{p} d \mu \leq C \delta^{p}\|\mu\|_{*} \int|f|^{p} d A
$$

Proof. The main idea appears in [3], but we will sketch a proof again here. First obtain the inequality

$$
\sup _{|z|<\eta / 2}|f(z)|^{p} \leq \frac{4}{\pi \eta^{2}} \int_{|\zeta|<\eta}|f(\zeta)|^{p} d A(\zeta) .
$$

This requires only the subharmonicity of $|f|^{p}$. If $f(0)=0$, apply this to $f(z) / z$ to get

$$
\begin{aligned}
|f(z)|^{p} & \leq \frac{4|z|^{p}}{\pi \eta^{2}} \int_{|\zeta|<\eta}\left|\frac{f(\zeta)}{\zeta}\right|^{p} d A(\zeta) \\
& \leq \frac{16|z|^{p}}{3 \pi \eta^{2}} \int_{\eta / 2<|\zeta|<\eta}\left|\frac{f(\zeta)}{\zeta}\right|^{p} d A(\zeta) \\
& \leq \frac{16|z|^{p}}{3 \pi \eta^{2}} \frac{2^{p}}{\eta^{p}} \int_{|\zeta|<\eta}|f(\zeta)|^{p} d A(\zeta) .
\end{aligned}
$$

The second inequality is again because the mean over $\{\eta / 2<|\zeta|<\eta\}$ exceeds the mean over $\{|\zeta|<\eta\}$. Then, for $|z|<\delta<\eta / 2$ we get

$$
|f(z)|^{p} \leq C\left(\frac{2 \delta}{\eta}\right)^{p} \frac{1}{\pi \eta^{2}} \int_{|\zeta|<\eta}|f(\zeta)|^{p} d A(\zeta)
$$

with $C=16 / 3$. Let $\sigma_{k}(z)=\left(z_{k}-z\right) /\left(1-\bar{z}_{k} z\right)$ and apply the above inequality to $f \circ \sigma_{k}$, where $f$ satisfies $f\left(z_{k}\right)=0$ to get

$$
\left|f\left(\sigma_{k}(z)\right)\right|^{p} \leq C\left(\frac{2 \delta}{\eta}\right)^{p} \frac{1}{\pi \eta^{2}} \int_{D_{\eta}\left(z_{k}\right)}|f(w)|^{p}\left|\sigma_{k}^{\prime}(w)\right|^{2} d A(w) .
$$

We now use the fact that for $w \in D_{\eta}\left(z_{k}\right)$ we have $\left|\sigma_{k}^{\prime}(w)\right| \leq\left(1+\left|z_{k}\right| \eta\right)^{2} /\left(1-\left|z_{k}\right|^{2}\right)$. Moreover, $\left|D_{\eta}\left(z_{k}\right)\right|=\pi \eta^{2}\left(1-\left|z_{k}\right|^{2}\right)^{2} /\left(1-\left|z_{k}\right|^{2} \eta^{2}\right)^{2}$, so we get

$$
\left|f\left(\sigma_{k}(z)\right)\right|^{p} \leq C\left(\frac{2 \delta}{\eta}\right)^{p} \frac{1}{\left|D_{\eta}\left(z_{k}\right)\right|}\left(\frac{1+\left|z_{k}\right| \eta}{1-\left|z_{k}\right| \eta}\right)^{2} \int_{D_{\eta}\left(z_{k}\right)}|f(w)|^{p} d A(w), \quad|z|<\delta .
$$

Now, $\sigma_{k}\left(D_{\delta}(0)\right)=D_{\delta}\left(z_{k}\right)$ and so

$$
|f(z)|^{p} \leq C \delta^{p} \frac{1}{\left|D_{\eta}\left(z_{k}\right)\right|} \int_{D_{\eta}\left(z_{k}\right)}|f(w)|^{p} d A(w), \quad z \in D_{\delta}\left(z_{k}\right),
$$

where $C=16\left[2^{p}(1+\eta)^{2}\right] /\left[3 \eta^{p}(1-\eta)^{2}\right]$ and depends only on $\eta$ and $p$. Integrate the last inequality with respect to $\mu$ on $D_{\delta}\left(z_{k}\right)$ and then sum on $k$ to get the inequality in the statement of the lemma.

We can use this lemma now to get a result like Lemma 2.2, but for a measure $\mu$ which need not be a vanishing Carleson measure.

Lemma 2.4. Let $\left\{z_{k}\right\}$ be a sequence of points such that the disks $D_{\eta}\left(z_{k}\right)$ are disjoint, and assume that $f \in A^{p}$ vanishes at all the points $z_{k}$. Let $\mu$ be a Carleson 
measure for $A^{p}$ which vanishes off the union $\bigcup_{k} D_{\delta_{k}}\left(z_{k}\right)$ for some sequence of pseudohyperbolic radii $\delta_{k}$ tending to 0 . Then

$$
\lim _{m \rightarrow \infty} \frac{\int\left|z^{m} f(z)\right|^{p} d \mu(z)}{\int\left|z^{m} f(z)\right|^{p} d A(z)}=0 .
$$

Proof. Fix $0<R<1$ and let $\mu=\mu_{1}+\mu_{2}$ where $\mu_{1}$ is concentrated on $|z|<R$ and $\mu_{2}$ is concentrated on $R \leq|z|<1$. Then $\mu_{2}$ satisfies the hypothesis of Lemma 2.3 for some $\delta=\delta(R)$ that tends to 0 as $R$ tends to 1 . Thus

$$
\frac{\int\left|z^{m} f\right|^{p} d \mu_{2}}{\int\left|z^{m} f\right|^{p} d A} \leq C\|\mu\|_{*} \delta^{p}
$$

and the right side can be made arbitrarily small by choosing $R$ near to 1 . Moreover, the argument in Lemma 2.2 can be used without change to show that

$$
\lim _{m \rightarrow \infty} \frac{\int\left|z^{m} f\right|^{p} d \mu_{1}}{\int\left|z^{m} f\right|^{p} d A}=0
$$

Combining these two observations yields the lemma.

It might be noted that a vanishing Carleson measure must fail to be 'bounded below' on any subspace of $A^{p}$ invariant under multiplication by $z$. Moreover, the measure $\mu$ in Lemma 2.4 must fail to be bounded below on the special invariant subspace of functions vanishing on the sequence $\left\{z_{k}\right\}$. This paper will not make any use of these observations.

\section{The CONSTRUCtion of $\varphi$}

The idea of the construction is to create a set and a 'counting function' $n_{\varphi}$ on it so that the measure $n_{\varphi} d A$ is the sum of two measures satisfying the hypotheses of Lemmas 2.2 and 2.4. As a first approximation we construct a zero sequence for $A^{2}$ which is also an $(\eta, R)$-lattice, and surround each point in that sequence with a pseudohyperbolic disk, the radii going to 0 as required by Lemma 2.4

There are several ways to create such a lattice, but a very concrete example can be created by applying a theorem from the paper [5].

Proposition 3.1. Let $\left\{z_{n k}: n=1,2,3, \ldots, k=1,2, \ldots, 8^{n}\right\}$ satisfy $1-\left|z_{n k}\right|=$ $8^{-n}$, the points $z_{n 1}, z_{n 2}, \ldots, z_{n 8^{n}}$ being equally spaced on the circle of radius $1-8^{-n}$. Then $\left\{z_{n k}\right\}$ is a zero sequence for $A^{2}$.

Proof. This is just Theorem 7 from [5] with $\beta=8, \gamma=1$ and $p=2$, the condition there being that $\gamma / \log \beta<1 / p$. This is clear because $\log 8>2$.

Straightforward estimates show that the circles with radii $1-8^{-n}$ are separated by a pseudohyperbolic distance at least $7 / 9$ but less than $7 / 8$. Moreover, very tedious estimates show that the points of $\left\{z_{n k}\right\}$ on a single such circle are separated by a pseudohyperbolic distance at least 0.89 , but less than 0.96 . Thus, we may crudely estimate that $\left\{z_{n k}\right\}$ is an $(\eta, R)$-lattice if $\eta<7 / 18$ and $R>367 / 368$. In the construction below, let such an $\eta$ and $R$ be fixed.

Let each $z_{n k}$ thus obtained be the center of a pseudohyperbolic disk $D_{n k}=$ $D_{\delta_{n}}\left(z_{n k}\right)$, where $\delta_{n} \leq \eta$, and $\delta_{n} \rightarrow 0$. If necessary, rotate the positions of the $z_{n k}$ 
so that none of the $D_{n k}$ intersect the positive real axis. For each $n$ choose an integer $M_{n}$ so that

$$
1<M_{n} \frac{\left|D_{n k}\right|}{\left|D_{\eta}\left(z_{n k}\right)\right|}<2 .
$$

Note that since the radii of $D_{n k}$ tend to 0 , we must have $M_{n} \rightarrow \infty$. The function $\varphi$ we will construct will have $n_{\varphi}=M_{n}$ on $D_{n k}$. This being the case, we will have $\int_{D_{\eta}\left(z_{n k}\right)} n_{\varphi} d A \geq M_{n}\left|D_{n k}\right|>\left|D_{\eta}\left(z_{n k}\right)\right|$, and this will lead to the lower bound (1.4). Moreover, if this were all there were to $n_{\varphi}$, we would also have $\int_{D_{\eta}\left(z_{n k}\right)} n_{\varphi} d A \leq$ $M_{n}\left|D_{n k}\right| \leq 2\left|D_{\eta}\left(z_{n k}\right)\right|$, and this leads to the required upper bound (1.1), because an arbitrary $D_{\eta}(z)$ meets at most two $D_{\eta}\left(z_{n k}\right)$.

Ideally, we would like the range of $\varphi$ to be the union of these disks, but an analytic function must have a connected range. Therefore, we connect the circle of disks $D_{n k}, 1 \leq k \leq 8^{n}$, with an annulus $A_{n}$, and then we connect these annuli together. These connections must be chosen to be very thin near the boundary of the unit disk, so that $n_{\varphi} d A$ will be a vanishing Carleson measure on these connecting pieces.

To this end, let $A_{n}$ be an annulus containing the circle of radius $1-8^{-n}$. The annuli are chosen to be so thin that they are disjoint, and so that if $\nu=\sum_{n} M_{n} \chi_{A_{n}} d A$, then $\nu$ is a vanishing Carleson measure. For this, it is sufficient to make the thickness of $A_{n}$ be $8^{-n} \epsilon_{n} / M_{n}$ with $\epsilon_{n} \searrow 0$. For then if $S_{h}$ is a Carleson square with sidelength $h$ we will have

$$
\nu\left(S_{h}\right)=\sum M_{n}\left|A_{n} \cap S_{h}\right| \sim \sum_{8^{-n} \leq h} M_{n} \frac{8^{-n} \epsilon_{n}}{M_{n}} h \leq \epsilon_{k} h \sum_{8^{-n} \leq h} 8^{-n} \leq C \epsilon_{k} h^{2},
$$

where $k$ is the first integer with $8^{-k} \leq h$. Our constructed $\varphi$ will have $n_{\varphi} \leq M_{n}$ on $A_{n}$.

Lastly, let $G$ be the domain in $\mathbb{D}$ bounded below by the $x$-axis and above by the curve $y=x(1-x)^{2}, 0 \leq x \leq 1$. The boundary of $G$ touches the boundary of the unit disk only at the point 1 , where it has a cusp. Therefore $\chi_{G} d A$ is a vanishing Carleson measure. Our $\varphi$ will satisfy $n_{\varphi}=1$ on $G \backslash\left(\bigcup_{n} A_{n} \cup \bigcup_{n, k} D_{n k}\right)$.

It is clear, if we succeed in constructing such a $\varphi$, that the measure $n_{\varphi} d A$ will satisfy the lower bound (1.4), because any pseudohyperbolic disk of radius $R^{\prime}$ exceeding $(R+\eta) /(1+R \eta)$ will contain one of the disks $D_{n k}$, and so

$$
\int_{D\left(z, R^{\prime}\right)} n_{\varphi} d A>\int_{D_{n k}} n_{\varphi} d A \geq D\left(z_{n k}, \eta\right)>\delta\left|D\left(z, R^{\prime}\right)\right|
$$

for some $\delta=\delta\left(R^{\prime}, \eta\right)>0$. Moreover, $n_{\varphi} d A$ is a Carleson measure (satisfies the upper bound (1.1) ) because it is composed of parts which have been required to satisfy that same condition. However, the restriction of the measure $n_{\varphi} d A$ to the union of those disks will satisfy the condition of Lemma 2.4, while its restriction to the union of the annuli $A_{n}$ and $G$ will satisfy the condition of Lemma 2.2. Thus it will not satisfy condition (2.3). Therefore, the function $\varphi$ will satisfy conditions (1.1) and (1.4), but the composition operator $C_{\varphi}$ will not have closed range. This $\varphi$ will be the promised example.

Let $W \stackrel{\text { def }}{=} \bigcup_{n, k} D_{n k} \cup \bigcup_{n} A_{n} \cup G$ and consider it's inverse image $\exp ^{-1} W$ under the exponential function. Naturally, the exponential function maps an infinite number of points in the left half plane to any given point in $W$. Therefore we discard enough of $\exp ^{-1} W$ so that the number of preimages of each point matches 
our desired $n_{\varphi}$. To this end, discard all but one copy of the preimage of $G$, keeping only the one that stands just above the negative real axis; discard all but $M_{n}$ copies of the preimage of each $D_{n k}$, keeping only those that lie between the $x$-axis and the line $\operatorname{Im} z=2 M_{n} \pi$. Finally, the preimage of each annulus $A_{n}$ is an infinite vertical strip; we keep only the points in each such strip with imaginary part between 0 and $2 M_{n} \pi$. Let $V$ denote the set of points of $\exp ^{-1} W$ that we have not discarded.

If we think of the preimage of $G$ as a hillside in the second quadrant, sloping gently down to the right, then the set $V$ consists of that hillside, plus an infinite number of tall, thin stalks (the preimages of the annuli $A_{n}$ ) growing up along the vertical lines $\operatorname{Re} z=\log \left(1-8^{-n}\right)$ to height $2 M_{n} \pi$ and bearing a large number $\left(8^{n} M_{n}\right)$ of equally spaced fruits (the preimages of the disks $\left.D_{n k}\right)$. We have arranged that the exponential function maps a given stalk with its fruits $M_{n}$-to-one onto the corresponding annuli and disks, and maps the rest of $V$ one-to-one onto the rest of $W$. The exponential function restricted to $V$ therefore has the same counting function as we want $\varphi$ to have.

The set $V$ is a simply connected domain. If we let $\psi$ be the conformal map from $\mathbb{D}$ onto this domain, then $\varphi=\exp \circ \psi$ will have the same counting function on $W$ as the exponential function restricted to $V$, so $\varphi$ is the desired mapping.

\section{REFERENCES}

[1] Eric Amar, Suites d'interpolation pour les classes de Bergman de la boule et du polydisque de $C^{n}$, Canad. J. Math. 30 (1978), 711-737. MR 58:17208

[2] M. Jovović and B. MacCluer, Composition operators on Dirichlet spaces, Acta Sci. Math. 63 (1997), 229-247. MR 98d:47067

[3] Daniel H. Luecking, Forward and reverse Carleson inequalities for functions in Bergman spaces and their derivatives, Amer. J. Math. 107 (1985), 85-111. MR 86g:30002

[4] , Dominating measures for spaces of analytic functions, Illinois J. Math. 32 (1988), 23-39. MR 89e:46060

[5] _ Zero sequences for Bergman spaces, Complex Variables Theory Appl. 30 (1996), 345-362. MR 97g:30007

[6] Richard Rochberg, Interpolation by functions in Bergman spaces, Michigan Math. J. 29 (1982), 229-236. MR 83e:30039

[7] N. Zorboska, Composition operators on weighted Dirichlet spaces, Proc. Amer. Math. Soc. 126 (1998), 2013-2023. MR 98h:47047

Department of Mathematical Sciences, University of Arkansas, Fayetteville, ArkANSAS 72701

E-mail address: luecking@comp.uark.edu

$U R L:$ http://comp.uark.edu/ luecking/ 